Aporte Santiaguino. 10 (1), 2017: 13-24

ISSN 2070-836X

\title{
Predictores del burnout académico en estudiantes de enfermería de las universidades nacionales de Áncash
}

\author{
Predictors of the academic burnout in nursing students Áncash national \\ universities \\ LLERMÉ NÚÑ̃EZ ZARAZÚ ${ }^{1}$
}

\section{RESUMEN}

Determina los predictores intrapersonales, sociales y académicas de las dimensiones del burnout académico; así como su prevalencia e índice en estudiantes de enfermería. Estudio tipo descriptivo transversal correlacional. La muestra censal estuvo conformada por 348 estudiantes de enfermería, de la Universidad Nacional Santiago Antúnez de Mayolo y la Universidad Nacional del Santa. El instrumento incluyó el cuestionario Maslach Burnout Inventory (MBI-SS) para estudiantes y un cuestionario sobre aspectos del estudiante (CUESESTE). La prevalencia del burnout académico fue de 7\% con índices medios de alta eficacia académica, agotamiento emocional medio y cinismo bajo. Predicen los índices de agotamiento emocional: la sobrecarga académica, la depresión y no realizar otras actividades extracurriculares; predicen los niveles de cinismo: ansiedad, dependencia alcohólica y depresión; predicen los niveles de eficacia académica: edad, funcionalidad familiar y vocación por la carrera. Se concluye que existe una baja prevalencia de síndrome de burnout en la población universitaria de enfermería, pero preocupan las variables intrapersonales, académicas y sociales que las predicen; las que deben definir las temáticas de tutoría y consejería docente, además de programas de prevención e intervención psicoeducativa.

Palabras clave: salud mental; burnout académico; universitarios; enfermería.

\begin{abstract}
It is determineds the intrapersonal, social and academic predictors of the dimensions of academic burnout; as well as its prevalence and index in nursing students. Descriptive cross-sectional descriptive study. The census sample consisted of 348 nursing students, from the National University Santiago
\end{abstract}

Universidad Nacional Santiago Antúnez de Mayolo. Huaraz, Perú. 
Antúnez de Mayolo and the National University of Santa. The instrument included the Maslach Burnout Inventory (MBI-SS) for students and a questionnaire on student aspects (CUESESTE). The prevalence of academic burnout was 7\%, with average rates of high academic efficacy, average emotional exhaustion and low cynicism. Predict rates of emotional exhaustion: academic overload, depression and not performing other extracurricular activities; predict levels of cynicism: anxiety, alcohol dependence, and depression; predict the levels of academic effectiveness: age, family functionality and vocation for the career. It is concluded that there is a low prevalence of burnout syndrome in the nursing university population, but the intrapersonal, academic and social variables that predict them are of concern; which should define the topics of tutoring and teaching, as well as prevention and psych educational intervention programs.

Keywords: mental health; burnout academic; university; nursing.

\section{INTRODUCCIÓN}

Las instituciones de educación superior suelen ser lugares altamente estresantes. En dichas instituciones los alumnos se enfrentan de manera cotidiana a una serie de demandas que, bajo su propia valoración, se constituyen en situaciones generadoras de estrés.

Desde una perspectiva psicosocial, Caballero, Bresó y Gonzales (2015) afirman que el universitario está expuesto a múltiples demandas que ejercen presión sobre su rol y desempeño académico, lo que puede dar sensación intensa de tensión, rechazo y fracaso del mismo; así como también cuando el estudiante no logra responder adaptativamente a los eventos de estrés, resolver sus demandas y emociones asociadas propias de la vida académica; puede entonces sentirse impedido y desmotivado cada vez más para resolver exitosamente estas situaciones, además de que estas aumenten acompañándose de un proceso acumulativo de malestar prolongado.

Cuando son prolongadas y frecuentes, estas situaciones de estrés pueden contribuir a generar sensaciones de no poder dar más de sí mismo, tanto física como psíquicamente, una actitud negativa de crítica, desvalorización, pérdida de interés de la trascendencia, del valor frente al estudio y dudas crecientes de poder realizarlo. La presencia simultánea de estas manifestaciones es conocida como síndrome de burnout académico. El estudio del síndrome ha tenido un amplio desarrollo en los últimos años, aportando a la comprensión de los procesos de estrés asistencial, organizacional y académico, con miras a mejorar la calidad de vida de los empleados y estudiantes. 
Justifica la presente investigación, el hecho de que en el Perú existen 31055 estudiantes de Enfermería, formándose en 57 Escuelas o Facultades de Enfermería públicas o privadas; constituyendo la quinta carrera con mayor número de alumnos matriculados a nivel del país. Además, los centros superiores de formación, actualmente inmersos en la autoevaluación y acreditación universitaria, hacen esfuerzos para mejorar la calidad educativa que ofrecen a los estudiantes; pero aún siguen soslayando el bienestar personal de sus educandos, a pesar que este constituye parte de la multicausalidad de deserción, bajo rendimiento o baja calidad de vida de este grupo poblacional.

El burnout académico afecta la salud mental de los estudiantes, causando disfunción mental en la medida que precipita depresión y/o ansiedad; también está relacionado con bajo desempeño académico, menores expectativas de éxito en los estudios e intención de abandono de los mismos, entre otras consecuencias negativas.

Los estudiantes de Enfermería son un grupo susceptible de tener problemas de burnout, debido a que se ven sometidos a una sobrecarga de actividades académicas en el aula, mezclada con prácticas clínicas o comunitarias, con el adicional de tareas académicas; todo lo cual configura una sobrecarga laboral.

En otras partes del mundo reportan niveles medios del burnout académico en estudiantes universitarios, siendo siempre mayores en alumnos de las ciencias humanas.

Por todo lo anterior, se plantean los siguientes objetivos en la investigación: identificar la prevalencia y los índices de las dimensiones del burnout académico en los estudiantes de enfermería de las universidades nacionales de Áncash $\mathrm{y}$; determinar los predictores intrapersonales, sociales y académicos de las dimensiones del burnout académico en los estudiantes de enfermería de las universidades nacionales de Áncash.

Las variables de estudio son: Burnout académico, con sus tres dimensiones (agotamiento emocional, cinismo y eficacia académica). Variables intrapersonales del estudiante: edad, género, número de hijos, consumo de sustancias psicoactivas, riesgo suicida, tabaquismo, estrés por compatibilizar trabajo y estudio, dependencia alcohólica, ansiedad, depresión, estrés académico, expectativa de éxito. Variables sociales del estudiante: trabajo, actividades extracurriculares, estado civil, disponibilidad económica para estudios, conformidad con su situación económica, funcionalidad familiar. Variables académicas del estudiante: año académico, número de cursos matriculados, prácticas clínicas/ comunitarias/laboratorios, percepción académica, satisfacción del rendimiento académico, sobrecarga académica, tendencia al abandono de estudios, vocación por la carrera, desempeño académico. 


\section{MATERIALES Y MÉTODOS}

El presente estudio fue de tipo descriptivo, de diseño no experimental y de corte transversal. La población de estudio estuvo compuesta por los 348 estudiantes de Enfermería matriculados en el semestre académico 2014-I de las universidades: Universidad Nacional Santiago Antúnez de Mayolo (UNASAM) y de la Universidad Nacional del Santa (UNS), del departamento de Áncash - Perú. La muestra fue de carácter censal por el bajo número poblacional.

Los criterios de inclusión fueron: ser estudiantes universitarios matriculados en la escuela de Enfermería de una universidad pública de Áncash y aceptar voluntariamente cumplimentar el cuestionario.

Como instrumento de recolección de datos se utilizó un cuestionario denominado "Cuestionario del estudiante de enfermería" (CUESEST) el cual incluía ítems sobre la sociodemografía de la unidad muestral y algunos test estandarizados como el MBI-SS (Gil-Monte, 2001) en su versión española, denominado Inventario para evaluar el síndrome de Burnout para estudiantes. El MBI-SS estuvo compuesto por 15 ítems divididos en tres dimensiones: agotamiento emocional (5 ítems), despersonalización o cinismo (4 ítems) y eficacia académica (6 ítems). Los ítems fueron respondidos según una escala tipo Likert de seis puntos (de 0 Nunca a 6 todos los días), donde las altas puntuaciones en agotamiento emocional y despersonalización, y bajas puntuaciones en eficacia personal serían indicadores del burnout académico.

Para la confiabilidad del MBI-SS durante su estandarización en la población española obtuvo valores confiables y en este estudio también los valores encontrados fueron aceptables porque superaron el 0.70 recomendado. Se halló un alpha de Cronbach de 0.792 para la escala de agotamiento emocional, 0.739 para la escala de cinismo y un valor de 0.805 para la escala de eficacia académica.

Para la validez del MBI_SS, durante su estandarización en población española, la escala demostró validez y adicionalmente en el estudio se realizó un análisis factorial confirmatorio, obteniendo valores aceptables para el IFI $=0.845$, $\mathrm{NFI}=0.822, \mathrm{CFI}=0.844$ y GFI $=0.813$, lo cual confirmó que los ítems responden a las dimensiones que dicen medir.

Se tuvo como consideraciones éticas: La participación de los estudiantes fue voluntaria y anónima, con consentimiento informado previo, no se realizó ninguna codificación que pudiera identificar a los participantes y la información recolectada se utilizó solo para los fines de la investigación. 


\section{RESULTADOS}

\section{Características sociodemográficas}

La edad de los universitarios del estudio de investigación, tuvo una media de 20.49, una mediana de 20.00 y un rango de 16 años; siendo para las mujeres la media de $20.5(\mathrm{~N}=311$, rango de 16 a 31 y mediana de 20.5) y para los varones una media de $20.41(\mathrm{~N}=37$, rango de 17 a 26 y mediana de 20.00).

Las características de la población estudiada en general fueron las siguientes: el $89.4 \%$ pertenecieron al género femenino, el $61.8 \%$ se encontró dentro del grupo etáreo de adulto joven, el $51.4 \%$ estudió en la Universidad Nacional del Santa; el $94.5 \%$ presentó como estado civil la soltería, el 93.1\% no tuvo hijos, el 34.5\% vivió en una familia nuclear y el 76.4 no trabajó, solamente estudió.

\section{Burnout académico}

Los valores generales en las dimensiones del burnout académico revelaron altos índices de eficacia académica $(\mathrm{M}=4.06 ; \mathrm{DE}=1.07)$, nivel medio de agotamiento emocional $(\mathrm{M}=1.98 ; \mathrm{DE}=1.05)$ y bajos índices de cinismo $(\mathrm{M}=1.39$; $\mathrm{DE}=1.00)$. Ver tabla 1 .

Así mismo se encontró que el $6.9 \%$ de universitarios presentó altos niveles de agotamiento emocional, altos niveles de cinismo y bajos niveles de eficacia académica; conglomerado que precisa la definición del burnout académico.

Tabla 1. Medias y desviaciones estándar de las dimensiones del burnout académico en estudiantes de enfermería de las universidades nacionales de Áncash 2014

\begin{tabular}{ccc}
\hline Dimensiones & Media & DS \\
\hline Agotamiento emocional & 1.98 & 1.05 \\
Cinismo & 1.39 & 1.00 \\
Eficacia académica & 4.01 & 1.07 \\
\hline
\end{tabular}

El análisis de varianza realizado considerando como factores universidad y sexo (tabla 2) mostró que los estudiantes de la universidad de la costa y la sierra de Áncash difirieron poco en los puntajes obtenidos en las dimensiones del burnout académico. Son los estudiantes de la UNASAM los que presentaron un perfil con puntuaciones ligeramente más elevadas en cinismo, y más bajas en eficacia académica y agotamiento emocional, en relación a sus pares de la UNS, pero no significativas estadísticamente. 
Tabla 2. Análisis de varianza de las dimensiones del burnout académico en función de variables sociodemográficas en estudiantes de enfermería de las universidades nacionales de Áncash 2014

\begin{tabular}{|c|c|c|c|c|c|c|}
\hline & \multicolumn{3}{|c|}{ Género } & \multicolumn{3}{|c|}{ Universidad } \\
\hline & Hombre & Mujer & $\mathrm{F}$ & UNASAM & UNS & $\mathrm{F}$ \\
\hline $\begin{array}{l}\text { Agotamiento } \\
\text { emocional }\end{array}$ & 2.14 & 1.96 & 1.020 & 1.90 & 2.05 & 1.657 \\
\hline Cinismo & 1.67 & 1.35 & 3.339 & 1.41 & 1.36 & 0.350 \\
\hline Eficacia académica & 3.77 & 4.03 & 1.974 & 3.97 & 4.04 & 0.215 \\
\hline
\end{tabular}

Cuando se tiene como factor la universidad en el mismo tipo de análisis de varianza, fueron los estudiantes de la UNASAM los que obtuvieron los peores resultados en el mayor consumo de sustancias psicoactivas, mayor riesgo suicida y peor desempeño académico; mientras que los estudiantes de la UNS obtuvieron el mayor estrés académico.

En el análisis de varianza teniendo como factor el sexo, los resultados han sido favorables en su mayoría a los varones. Estos han mostrado estar menos estresados académicamente, tener mayor vocación por la carrera que estudian, pero realizar menos actividades extracurriculares que las mujeres.

Predictores del burnout académico

Para identificar las variables intrapersonales, sociales y académicas que predicen mayores índices de agotamiento, cinismo y eficacia académica en los estudiantes Enfermería, se realizó un análisis de regresión lineal múltiple para cada dimensión que comprende el burnout académico en el MBI-SS. En los tres análisis de regresión lineal múltiple, las variables independientes fueron las mismas variables intrapersonales, sociales y académicas.

En la tabla 3 se presentan las variables que predijeron las dimensiones del burnout académico en el MBI-SS (agotamiento emocional, cinismo y eficacia personal). 
Tabla 3. Regresión lineal múltiple con dimensiones del burnout académico como variable dependiente, en estudiantes de enfermería de las universidades nacionales de Áncash 2014

\begin{tabular}{lcccc}
\hline $\begin{array}{l}\text { Variables } \\
\text { Agotamiento emocional }\end{array}$ & $\mathrm{B}$ & Beta & $\mathrm{t}$ & $\mathrm{p}$ \\
\hline Sobrecarga académica & 1.785 & 0.606 & 3.792 & 0.000 \\
Depresión & 0.377 & 0.206 & 2.516 & 0.014 \\
Actividades extracurriculares & -2.086 & -0.290 & -2.323 & 0.023 \\
& & & & \\
Cinismo & & & & \\
$\quad$ Ansiedad & 0.247 & 0.326 & 1.697 & 0.004 \\
$\quad$ Dependencia alcohólica & 1.003 & 0.228 & 2.651 & 0.11 \\
Depresión & 0.442 & 0.407 & 2.222 & 0.31 \\
& & & & \\
Eficacia académica & & & & \\
Edad & 0.937 & 0.790 & 13.251 & 0.000 \\
Funcionalidad familiar & 2.666 & 0.261 & 4.994 & 0.000 \\
$\quad$ Vocación por la carrera & 1.470 & 0.086 & 2.653 & 0.008 \\
\hline
\end{tabular}

Los resultados indicaron que la sobrecarga académica, los altos puntajes de depresión y el no realizar otras actividades extracurriculares, predijeron los índices de agotamiento $(\mathrm{F}=125.425 ; \mathrm{p}<0,000 ; \mathrm{R}=0.868)$, lo cual explicó el 86.8\% del fenómeno.

Los puntajes de ansiedad, de dependencia alcohólica y los puntajes de depresión predijeron los niveles de cinismo ( $\mathrm{F}=58.932$; $\mathrm{p}<0.000 ; \mathrm{R}=0.763)$, lo cual explicó el $76.3 \%$ del fenómeno.

La edad, la funcionalidad familiar y la vocación por la carrera predijeron los niveles de eficacia académica $(F=1302.765$; $\mathrm{p}<0.000 ; \mathrm{R}=0.935)$, lo cual correspondió a un $93.6 \%$ del fenómeno.

\section{DISCUSIÓN}

El estudio del burnout académico, es un área de investigación reciente en el mundo y nuevo en nuestro país. Los estudios de este tipo han estado direccionados a confirmar su presencia en estudiantes universitarios como grupo preprofesional.

El inventario de Burnout de Maslach para estudiantes (MBI-SS) se utiliza como instrumento para medir el burnout académico porque ha demostrado, en diversos estudios, altos niveles de confiabilidad y validez; nuevamente ahora ha presentado valores aceptables en cada dimensión estudiada (agotamiento emocional, cinismo y eficacia académica). 
La población de estudiantes de enfermería tiene un rango de edad, que comprende el final de la adolescencia y al adulto joven, subetapas que coinciden paralelamente con los estudios de educación superior técnica o universitaria en nuestro país.

Las características sociodemográficas de la población: sexo, grupo etáreo, estado civil, número de hijos, con quién reside, trabajo y año de estudios fueron homogéneas para las dos universidades en estudio (UNASAM y UNS).

En relación a la presencia del burnout académico, se encuentra que el 7\% de la población lo presenta en su constructo tridimensional, según la definición de Schaufeli y otros (2002): alto nivel de agotamiento emocional y cinismo junto a un bajo nivel de eficacia académica. Este hallazgo de prevalencia en el estudio, contrasta con los encontrados con valores de $0 \%$ en Cuba (Barco y otros, 2008) y Brasil (Tomasshewski-Barlem y otros, 2014), 18.4 \% en México (Velasco, Córdova y Suarez, 2013) y 58\% en Israel (Acioli y Beresin, 2007). En estos estudios también utilizaron el MBI-SS, pero en la presentación de sus resultados no describieron detalladamente cómo definieron los casos de burnout, por lo que esta comparación debe ser tomada discretamente.

En el estudio, los estudiantes de Enfermería presentan altos índices de eficacia académica, índices medios de agotamiento emocional y bajos índices de cinismo. Resultados en su mayoría semejantes a los hallados en tres universidades de Brasil (Carlotto, Nakamura y Goncalves, 2006), también en España y Portugal (Martínez y Marques, 2005), con la diferencia que en una muestra en Brasil el índice de agotamiento emocional fue alto y en España-Portugal el índice de eficacia fue medio.

Cuando son comparados los estudiantes de las dos universidades de estudio, en relación a las tres dimensiones del burnout académico, se observa que sus puntuaciones medias fueron muy semejantes con ligeras variaciones menores a la mitad de la unidad; lo cual nos evidencia que a pesar de que proceden de dos regiones naturales diferentes del mismo departamento no varían significativamente.

Algo parecido ocurrió al comparar los índices de las dimensiones del burnout académico según sexo, no hallamos diferencias significativas. Hallazgos parecidos fueron reportados en estudiantes de Odontología y Medicina. Este último resultado no coincide con estudios en ámbitos laborales, donde los varones evidenciaron mayores niveles de cinismo, lo que dificultaría su desempeño laboral-social y la relación con sus congéneres (Salanova y Llorens, 2008).

Cuando comparamos todas las otras variables estudiadas según la universidad de procedencia, son significativas las diferencias para algunas variables. Así los estudiantes de la UNASAM, presentan peores resultados para el consumo de sustancias psicoactivas, el desempeño académico y el riesgo suicida; mientras 
que los estudiantes de la UNS presentan más estrés académico. Estas diferencias se deben probablemente a que cada universidad organiza sus estudios de manera diferente, considerando la libertad de cátedra de los profesores para planificar su asignatura, en lo que respecta a lo académico y en lo social cada universidad pertenece a regiones naturales diferentes y por ende su cultura y costumbres también son diferentes.

Al comparar las variables estudiadas según sexo, se hallan diferencias significativas en algunas de ellas. Así las mujeres se muestran más estresadas por asuntos académicos y realizan mayores actividades extracurriculares. Por otro lado, los varones muestran tener mayor vocación por la carrera de Enfermería. Estas diferencias de género coinciden en parte con la de estudiantes de Enfermería de Portugal donde las mujeres evidenciaron un mayor nivel de estrés que los hombres (Alves, Alves y Pineda, 2007). Además, siendo Enfermería históricamente una carrera predominantemente femenina, se puede deducir que los hombres que eligen estudiar esta profesión lo hacen porque presentan un alto grado vocacional.

En relación con la dimensión agotamiento, se observa que tener más sobrecarga académica, tener altos puntajes de depresión y realizar menos o ninguna actividad extracurricular son factores generadores de desgaste emocional. Es importante resaltar que percibir como estresante la sobrecarga académica es el indicador intrapersonal con mayor poder predictivo para el agotamiento emocional. Este resultado confirma que la mayor parte de los factores de esta dimensión son derivadas de situaciones de sobrecarga. El agotamiento emocional se relaciona de manera general, con exigencias excesivas que provienen del ejercicio del trabajo. La sobrecarga laboral es un predictor del agotamiento emocional y en el caso de los estudiantes serán la cantidad de actividades teóricas y prácticas que realizan (Maslach, 1982).

Brallan y otros (2009) en Colombia coincide en que influyen en la presencia del síndrome de burnout académico en este grupo de estudio la sobrecarga académica impuesta al estudiante dado por sobrecarga de tareas académicas en tiempos limitados, número alto de créditos, evaluaciones abundantes de los profesores e intensidad horaria.

En lo relacionado al cinismo, sus variables predictoras fueron: mayores puntajes de ansiedad, mayor dependencia alcohólica y mayor puntaje de depresión; siendo la mayor ansiedad el factor con mayor poder explicativo. Estos resultados difieren a los hallados en un grupo de estudiantes de un curso técnico de Enfermería en Brasil, donde las variables predictoras para cinismo fueron la falta de retroalimentación positiva y el estrés percibido en la relación con los otros colegas del curso (Carlotto, Nakamura y Goncalves, 2006). 
Observamos que las tres variables predictoras del cinismo son de carácter intrapersonal y específicamente de salud mental. Esto preocupa y necesita intervenciones urgentes que mejoren la salud mental de los preprofesionales de la salud en general y de enfermería en particular.

En referencia a la dimensión de eficacia académica o competencia percibida, se verifica que tener menor edad, peor funcionalidad familiar y poca vocación por la carrera ocasiona sentimientos de falta de eficacia académica. La sensación de sobrecarga, producida por demandas de la familia y del contexto académico, dificulta la realización adecuada de las tareas y disminuyen así los niveles de autoeficacia general del sujeto, lo que engloba también la percepción de su desempeño laboral y académico (Beas y Salanova, 2001). Otros estudios coinciden en que la funcionalidad familiar es un factor importante para la eficacia académica (Opazo, Pino y Potthoff, 2012).

Como limitaciones en el presente estudio se tiene la técnica de recolección de datos, el aplicar el instrumento en grupo puede llevar a la falta de sinceridad en las respuestas de los estudiantes en temas como el uso de sustancias psicoactivas, desempeño académico, riesgo suicida, etc.

\section{CONCLUSIONES}

El 7\% de estudiantes de enfermería presentan burnout académico, es decir, altos niveles de agotamiento emocional, altos niveles de cinismo y bajos niveles de eficacia académica. Así mismo, la mayoría de los estudiantes de enfermería presentan altos índices de eficacia académica, índices medios de agotamiento y bajos índices de cinismo. Finalmente se hallaron como predictores de los índices de agotamiento emocional: la sobrecarga académica, los altos puntajes de depresión y el no realizar otras actividades extracurriculares. Predicen los niveles de cinismo: los puntajes de ansiedad, la dependencia alcohólica y los puntajes de depresión. Y son predictores de los niveles de eficacia académica: la mayor edad, la funcionalidad familiar y la vocación por la carrera. Se espera que los predictores encontrados, contribuyan a planificar la consejería-tutoría académica, gestionar cambios académicos y promover acciones de bienestar universitario.

\section{REFERENCIAS BIBLIOGRÁFICAS}

Acioli, Juliana y Beresin, Ruth. 2007. «Burnout syndrome in nursing undergraduate students». Revista Einstein, Vol.5, № 3. Sao Paulo: Hospital Israelita. 225-230. 
Alves, Joao; Alves, Manuel y Pineda, Juan. 2007. «Evaluación de los estados emocionales de estudiantes de Enfermería». Index de Enfermería. Vol 16, $\mathrm{N}^{\circ}$ 56. Portugal: Unidad Investigación Ciencias de la Salud. 26-29.

Barco, Vladimir y otros. 2008. «El Síndrome de Burnout en enfermeros básicos del segundo año de carrera». Revista de Enfermería. Vol. 24, No 3. Cienfuegos: Facultad de Ciencias Médicas. 11-21.

Beas, María y Salanova, Marisa. 2001. «Autoeficacia y su relación con el bienestar psicológico y la formación con los ordenadores en usuarios de CA». Simposium sobre autoeficacia: Investigación y Aplicaciones; Castellon: Universitat Jaume I.3-8

Brallan, Erika y otros. 2009. «Síndrome de burnout en estudiantes de enfermería en tres instituciones de educación superior, Cartagena de Indias». Ciencia y Salud, Vol. 1, No 1. Cartagena: Corporación Universitaria Rafael Nuñez. 643-655.

Caballero, Carmen; Bresó, Edgar y Gonzales, Orlando. 2015. «Burnout en estudiantes universitarios». Psicología desde el Caribe, Vol. 32, № 3. Barranquilla: Universidad del Norte. 424-441.

Carlotto Mary; Nakamura, Antonieta y Goncalves, Sheila. 2006. «Síndrome de Burnout em estudantes universitarios da área da saudé». Psico, Vol. 37, $\mathrm{N}^{\circ}$ 1. Porto Alegre: Universidad Luterana del Brasil. 57-62.

Gil-Monte, Pedro. 2001. «Validez factorial de la adaptación al español del Maslach Burnout Inventory-General Survey». Revista de Salud Pública de México. Vol 44, No 1. Cuernavaca: INSP.33-40.

Martínez, Isabel y Marques, Alexandra. 2005. «Burnout en estudiantes universitarios de España y Portugal y su relación con variables académicas». Aletheia. $\mathrm{N}^{\mathrm{o}}$ 21. Castellón: Universitat Jaume I. 21-30.

Maslach, Cristina. 1982. Burnout: the cost of caring. New Jersey: Prentice Hall.

Opazo, Cristina; Pino, Karla y Potthoff, María. 2012. Factores personales, familiares y laborales asociados al Sindrome de Burnout en el personal de Enfermería. Valdivia: Hospital de Valdivia.

Salanova, Marisa y Llorens, Susana. 2008. «Estado actual y retos futuros en el estudio del Burnout». Papeles del Psicólogo, Vol. 29, No 1. Madrid: Consejo General de Colegios Oficiales de Psicólogos. 59-67.

Schaufeli, Wilmar y otros. 2002. «Burnout and engagement in university students: across national study». Journal of Cross-Cultural Psychology, Vol. 33, $\mathrm{N}^{\circ}$ 5. Washington: Western Washington University. 464-481. 
Tomasshewski-Barlem, Jamile y otros. 2014. «Síndrome de burnout entre estudiantes de pregrado en enfermería de una universidad pública». Revista Latino-Am Enfermagem, Vol. 22, Nº 6. Río Grande: Universidad Río Grande. $8-16$.

Velasco, Víctor; Córdova, Sonia y Suarez, Gerardo. 2013. "Síndrome de desgaste estudiantil (Burnout) y su asociación con ansiedad y depresión en alumnos de una Escuela de Enfermería". Revista Iberoamericana para la investigación y

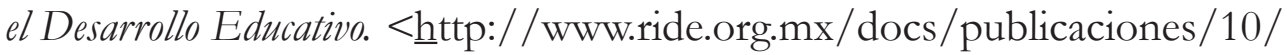
salud/B04.pdf> [consulta: 12-10-2015].

Presentado: $25 / 01 / 2017$

Aceptado: 28/04/2017

\section{Correspondencia}

Llermé Núñez Zarazú

opebri@yahoo.com 